\title{
The continued evolution of peripartum anesthesiologists
}

\author{
Won Lee, MD, ScM • Ronald B. George, MD, FRCPC
}

Received: 30 October 2021/Revised: 15 November 2021/Accepted: 15 November 2021/Published online: 22 December 2021

(C) Canadian Anesthesiologists' Society 2021

The scope of obstetric anesthesia continues to evolve beyond the confines of a labour and delivery suite. The traditional focus of obstetric anesthesia has been the administration of safe and effective labour analgesia and anesthetic care for Cesarean deliveries. These evidenceinformed efforts include ensuring safe and effective neuraxial catheters, minimizing maternal hypotension with pre-emptive use of vasopressors, using low concentrations of local anesthesia in labour epidurals to minimize motor blockade and reduce instrumental vaginal deliveries, and developing protocols to address obstetric emergencies like hemorrhage and pre-eclampsia. These achievements have reduced direct and indirect anesthetic causes of maternal mortality at a significantly greater rate than the overall reductions in maternal mortality. ${ }^{1,2}$ In the latest annual report of the Confidential Enquiry into Maternal Deaths and Morbidity in the United Kingdom (UK) and Ireland, which includes data on women who died during or up to one year after pregnancy between 2015 and 2017 , there was only one maternal death associated with anesthesia. $^{3}$ As the counterpart of the perioperative physician, the peripartum anesthesiologist can optimize care for pregnant patients during their whole peripartum period. $^{4}$

The demographics of parturients are evolving and coexisting medical conditions are more frequent, presenting additional challenges to safely managing the peripartum period. As directly-related anesthesia mortality is decreasing, pregnancy-related infections and sepsis are leading direct causes of maternal deaths. ${ }^{5}$ Pregnant women

W. Lee, MD, ScM $(\varangle) \cdot$ R. B. George, MD, FRCPC Department of Anesthesia and Perioperative Care, University of California San Francisco, San Francisco, CA, USA

e-mail: won.lee@ucsf.edu are predisposed to developing infections and sepsis because of significant hemodynamic and immunologic changes, increased metabolic demand, and decreased functional reserve. Anatomical changes can increase the risk of pyelonephritis and chorioamnionitis. The physiologic alterations in pregnancy can make the diagnosis of sepsis challenging and alter the effectiveness of many parameters used in the screening and diagnosis of sepsis in the general population.

In this month's issue of the Journal, Balki et al. ${ }^{6}$ present their findings on maternal mortality related to sepsis/septic shock in Canada. Between 2004 and 2017, the incidence of sepsis during deliveries in Canadian hospitals was 114 per 100,000 hospitalizations, with a mortality rate of $0.5 \%$. One in seven (14\%) women with sepsis in this cohort experienced significant sepsis-related morbidity. Among women with severe sepsis, $61 \%$ had organ failure and $78 \%$ required intensive care unit admission. The rate of sepsis had significant regional variability, with women from the Territories facing close to three times the risk of developing sepsis during their hospital stay (224 per 100,000) compared with women from New Brunswick.

Sepsis remains a significant concern globally, with an estimated global maternal death rate of 211 per 100,000 live births in 2017. This represents close to $10 \%$ of deaths among women of reproductive age that are due to maternal causes. $^{7}$ Maternal sepsis is not limited to developing nations. Sepsis is the third leading cause of pregnancyrelated deaths in the USA, where between 2001 and 2010, maternal mortality consistently rose by an average of $9 \%$ per year. ${ }^{8}$ The increase in sepsis-related maternal mortality in high-income nations may reflect the changing demography of the pregnant population.

A larger proportion of women are becoming pregnant at an advanced maternal age, with higher rates of health 
comorbidities like diabetes, hypertension, cardiac disease, and obesity. Medical advancements have also allowed women with congenital diseases to give birth, who previously would not have lived to child-bearing age. In vitro fertilization technology and availability of fetal medicine has increased the prevalence of high-risk pregnancies as well. ${ }^{9}$ In Balki et al.'s study, the odds of developing sepsis was 1.2 times higher for women who were $35 \mathrm{yr}$ or older. Likewise, women with history of chronic renal disease (odds ratio [OR], 8.6; 95\% confidence interval [CI], 6.7 to 11.1), cerebrovascular disease (OR, 8.0; $95 \% \mathrm{CI}, 4.3$ to 14.6$)$, liver disease (OR, $6.2 ; 95 \% \mathrm{CI}$, 4.4 to 8.9$)$, valvular heart disease (OR, $6.2 ; 95 \% \mathrm{CI}, 4.1$ to 9.4) and diabetes mellitus (OR, 1.5; 95\% CI, 1.1 to 2.2 ) had a significantly increased risk of developing sepsis during their pregnancy.

With increasing prevalence of high-risk pregnancies, many obstetric anesthesia divisions have implemented antenatal anesthesia clinics. Many patients require a multidisciplinary plan with obstetricians and neonatologists partnering with anesthesiologists, including those with pre-existing hereditary coagulopathies, cardiopulmonary anomalies, and history of anticoagulation therapy. Antenatal evaluation affords adequate time to develop optimal anesthetic plans prior to labour and delivery dates, and in turn helps to reduce unplanned general anesthesia for Cesarean deliveries. In Balki et al.'s cohort, women who underwent general anesthesia had a significantly higher risk of developing sepsis compared with neuraxial anesthesia for Cesarean deliveries (OR, 10.7; 95\% CI, 9.3 to 12.4) - and especially those who underwent emergent compared with elective Cesarean deliveries (OR, 2.9; $95 \%$ CI, 2.7 to 3.2).

Optimal management and prevention of puerperal sepsis requires a similar multidisciplinary approach, where anesthesiologists can play a significant role in designing and implementing protocols for early recognition and management of maternal sepsis. Once suspected, sepsis management requires early volume resuscitation and appropriate antibiotic therapy. Continuous monitoring and provision of supportive care helps to minimize organ dysfunction and risk of shock and death. Each hour of delay in administrating antibiotic therapy following sepsisrelated hypotension is associated with decreased survival of $7.6 \% .^{10}$ Establishing a Maternal Early Warning system (MEWS), which tracks variables such as vital signs and laboratory results, can facilitate timely escalation of necessary care to minimize sepsis-related morbidity and mortality. For diagnosis of maternal sepsis, MEWS has a higher sensitivity of than Quick Sequential Organ Failure assessment (2\% vs 50\%) and higher specificity than the Systemic Inflammatory Response Syndrome criteria (87\% vs 63\%), two tools used to diagnose sepsis and identify patients with poor outcomes. ${ }^{11}$
A standardized MEWS, like obstetric patient safety bundles, provides an evidence-based framework to guide clinicians in safely managing obstetric patients. The emphasis is on preparation (i.e., assessing risk, performing in situ drills and condition-specific protocols, and developing systems for addressing early warning signs), responding properly to and reporting impending maternal events, and a commitment to continually improving the system itself. ${ }^{12}$ The framework can be adapted to both high and low resource settings. Low-resource hospitals can implement feasible components and have processes in place for appropriate transfer to higher resource centres if need arise.

Appropriate resource utilization, proper triage of patients by clinical needs, and thus provision of optimal hospital care is essential for improving maternal outcomes and reducing maternal health disparities. In 2015, the American College of Obstetricians and Gynecologists (ACOG) and the Society for Maternal-Fetal Medicine proposed an obstetric hospital classification system based on availability of clinical staff and resources to facilitate patient triage based on maternal morbidity and mortality risk. ${ }^{13}$ In a review of the 2014 State Inpatient Database from the Healthcare Cost and Utilization Project and American Hospital Association Annual Survey from seven states, $2.4 \%$ of women overall delivered at hospitals without the appropriate level of maternal health resources and $43 \%$ of women who required a level III or IV centre delivered at an inappropriate centre, which may include ill-equipped anesthesia services. ${ }^{14}$ To fulfill the ACOG's requirement for level III (subspecialty care) and level IV (regional perinatal centres), "board-certified anesthesiologists with special training or experience in obstetrics in charge of obstetric anesthesia service" are necessary, with anesthesia service available at all times.

To address perceived areas of quality improvement, the Society of Obstetric Anesthesia and Perinatology have developed a designation of Centers of Excellence program for obstetric anesthesia care. This designation is to recognize institutions and programs that show excellence in obstetric anesthesia and provide a broad surrogate quality metric of institutions providing obstetric anesthesia care. ${ }^{15}$ The designation ensures that these centres have appropriate policies for staffing, equipment, protocols, and policies for dealing with obstetric emergencies and provide simulation and team trainings, quality assurance, and patient follow-up systems. The expanded role of anesthesiologists caring for women during childbirth could not have been more evident than during these past 18 months during the COVID-19 pandemic. Our involvement in a multidisciplinary approach to developing protocols for safe management of obstetric patients with COVID-19 became crucial. Anesthesiologists were at the forefront in developing protocols to address both infected and non-infected patients, accounting for delivery of 
labour analgesia, Cesarean anesthesia, and managing any complications including sepsis or postpartum hemorrhage. Obstetric anesthesiologists have a unique set of skills to provide safe and optimal care during the pandemic. ${ }^{16}$ The expertise of managing airway and hemodynamics, establishing airway access and other invasive monitoring, and administering blood transfusion and other life-saving interventions are paramount to reducing maternal mortality and morbidity. Coordination of care and development of the protocols by collaborating with obstetricians, neonatologists, critical care and infectious disease experts, and environmental health services, allows the multidisciplinary team to provide optional and safe clinical care to pregnant patients while protecting healthcare workers. ${ }^{17}$

In summary, the role of obstetric anesthesiologists continues to expand beyond the scope of labour epidurals and anesthesia for Cesarean delivery. To care for new waves of obstetric patients with more complex medical histories, anesthesiologists have to be more than just consultants during the labour and delivery process. The days of monotonous repetition of neuraxial placements are over. Critical timely appraisals and ongoing partnership with other members of the peripartum care team is not only recommended but also necessary for anesthesiologists to evolve into peripartum physicians. We should aspire to be dedicated team members to optimize care for pregnant patients during their whole peripartum period.

\section{Les anesthésiologistes du péripartum : une évolution constante}

La portée de l'anesthésie obstétricale continue d'évoluer audelà des confins de la salle de travail et d'accouchement. Traditionnellement, l'objectif de l'anesthésie obstétricale a toujours été l'administration sécuritaire et efficace d'analgésiques pour l'analgésie du travail et de soins anesthésiques pour les accouchements par césarienne. Ces efforts se sont fondés sur des données probantes et comprennent le positionnement de cathéters neuraxiaux sécuritaires et efficaces, la minimisation de l'hypotension maternelle grâce à l'utilisation préventive de vasopresseurs, l'utilisation de faibles concentrations d'anesthésiques locaux dans les péridurales pour le travail obstétrical afin de minimiser le bloc moteur et réduire les accouchements vaginaux assistés, et l'élaboration de protocoles pour traiter les urgences obstétricales comme l'hémorragie et la prééclampsie. Ces prouesses ont permis de réduire les causes anesthésiques directes et indirectes de mortalité maternelle à un rythme nettement supérieur aux réductions globales de la mortalité maternelle. ${ }^{1,2}$ Dans le dernier rapport annuel de l'Enquête confidentielle sur les décès et la morbidité maternels au Royaume-Uni et en Irlande, qui comprend des données sur les femmes décédées pendant ou jusqu'à un an après la grossesse entre 2015 et 2017 , seul un décès maternel a été associé à l'anesthésie. ${ }^{3}$ Complémentant le rôle du médecin périopératoire, l'anesthésiologiste péripartum peut optimiser les soins des patientes enceintes pendant toute leur période péripartum. ${ }^{4}$

La démographie des parturientes évolue et les conditions médicales coexistantes sont plus fréquentes, ce qui ajoute des défis supplémentaires à la gestion sécuritaire de la période du péripartum. Alors que la mortalité directement liée à l'anesthésie diminue, les infections et le sepsis liés à la grossesse ont pris la tête des causes directes de décès maternels. ${ }^{5}$ Les femmes enceintes sont prédisposées à développer des infections et un sepsis en raison des changements hémodynamiques et immunologiques importants qu'elles vivent, ainsi que de l'augmentation des exigences métaboliques et de la diminution des réserves fonctionnelles. Les changements anatomiques peuvent augmenter le risque de pyélonéphrite et de chorioamnionite. Les altérations physiologiques pendant la grossesse peuvent rendre le diagnostic de sepsis difficile et altérer l'efficacité de nombreux paramètres utilisés pour le dépistage et le diagnostic du sepsis dans la population générale.

Dans ce numéro du Journal, Balki et coll. ${ }^{6}$ présentent leurs conclusions sur la mortalité maternelle liée au sepsis et au choc septique au Canada. Entre 2004 et 2017, l'incidence de sepsis pendant les accouchements dans les hôpitaux canadiens était de 114 pour $100 \quad 000$ hospitalisations, avec un taux de mortalité de $0,5 \%$. Une femme sur sept (14\%) atteinte de sepsis dans cette cohorte a développé une morbidité significative liée au sepsis. Parmi les femmes atteintes de sepsis sévère, $61 \%$ ont subi une défaillance d'organe et $78 \%$ ont dû être admises aux soins intensifs. Le taux de sepsis variait considérablement d'une région à l'autre, les femmes des Territoires faisant face à près de trois fois plus de risques de développer un sepsis pendant leur séjour hospitalier (224 pour 100 000) que les femmes du Nouveau-Brunswick.

Le sepsis demeure une préoccupation majeure à l'échelle mondiale, avec un taux mondial de mortalité maternelle estimé à 211 pour 100000 naissances vivantes en 2017. Cela représente près de $10 \%$ des décès chez les femmes en âge de procréer dus à des causes maternelles. ${ }^{7}$ Le sepsis maternel ne se limite pas aux pays en voie de développement. Le sepsis constitue la troisième cause de décès liés à la grossesse aux États-Unis où, entre 2001 et 2010, la mortalité maternelle a affiché une augmentation constante de $9 \%$ par an en moyenne. ${ }^{8}$ L'augmentation de la mortalité maternelle liée au sepsis dans les pays à revenu 
élevé pourrait être le reflet de l'évolution de la démographie de la population enceinte.

Une plus grande proportion de femmes sont enceintes à un âge maternel avancé, avec des taux plus élevés de comorbidités comme le diabète, l'hypertension, les maladies cardiaques et l'obésité. Les progrès de la médecine ont également permis aux femmes atteintes de maladies congénitales d'accoucher, elles qui n'auraient auparavant peut-être pas atteint l'âge de procréer. Les technologies de fécondation in vitro et la disponibilité de médicaments fœtaux ont également augmenté la prévalence des grossesses à haut risque. ${ }^{9}$ Dans l'étude de Balki et coll., les chances de développer un sepsis étaient 1,2 fois plus élevées chez les femmes de 35 ans ou plus. De même, les femmes ayant des antécédents de néphropathie chronique (rapport de cotes [RC], 8,6; intervalle de confiance [IC] à 95 $\%, 6,7$ à 11,1), de maladie cérébrovasculaire (RC, 8,0; IC 95 $\%, 4,3$ à 14,6), de maladie hépatique (6,2; IC $95 \%, 4,4$ à 8,9), de valvulopathie $(6,2$; IC $95 \%, 4,1$ à 9,4) et de diabète $(1,5 ;$ IC $95 \%, 1,1$ à 2,2) présentaient un risque significativement accru de développer un sepsis pendant leur grossesse.

Avec la prévalence croissante des grossesses à haut risque, plusieurs services d'anesthésie obstétricale ont mis en place des cliniques d'anesthésie prénatale. De nombreuses patientes ont besoin d'un plan multidisciplinaire élaboré avec des obstétriciens et des néonatologistes en partenariat avec des anesthésiologistes, notamment celles atteintes de coagulopathies héréditaires préexistantes, d'anomalies cardiopulmonaires ou présentant des antécédents de thérapie anticoagulante. L'évaluation prénatale donne suffisamment de temps pour mettre au point des plans d'anesthésie optimaux avant les dates de travail et d'accouchement, ce qui contribue à réduire le taux d'anesthésie générale non planifiée pour les accouchements par césarienne. Dans la cohorte de Balki et coll., les femmes qui ont reçu une anesthésie générale couraient un risque significativement plus élevé de développer un sepsis par rapport à celles recevant une anesthésie neuraxiale pour un accouchement par césarienne (RC, 10,7; IC $95 \%, 9,3$ à 12,4) - et tout particulièrement celles qui subissaient un accouchement par césarienne urgent plutôt que programmé (RC, 2,9; IC $95 \%, 2,7$ à 3,2).

La prise en charge et la prévention optimales du sepsis puerpéral nécessitent une approche multidisciplinaire similaire, dans laquelle les anesthésiologistes peuvent jouer un rôle majeur dans la conception et la mise en œuvre de protocoles de détection précoce et de prise en charge rapide du sepsis maternel. Une fois le sepsis suspecté, sa prise en charge nécessite une réanimation volumique précoce et une antibiothérapie adaptée. Le monitorage continu et la prestation de soins de soutien aident à minimiser le dysfonctionnement des organes et le risque de choc et de décès. Chaque heure de retard dans l'administration de l'antibiothérapie après une hypotension liée au sepsis est associée à une diminution de la survie de
7,6 \%. ${ }^{10}$ La mise en place d'un système d'alerte maternelle précoce (soit le système MEWS - Maternal Early Warning System), qui suit des variables telles que les signes vitaux et les résultats de laboratoire, peut faciliter la mise en œuvre rapide des soins nécessaires pour minimiser la morbidité et la mortalité liées au sepsis. Pour le diagnostic du sepsis maternel, le système MEWS possède une sensibilité plus élevée que l'évaluation séquentielle rapide des défaillances d'organes (Quick Sequential Organ Failure assessment) (2\% vs $50 \%$ ) et une spécificité supérieure aux critères du Syndrome de réponse inflammatoire systémique (Systemic Inflammatory Response Syndrome criteria) (87\% vs 63 $\%$ ), deux outils utilisés pour diagnostiquer le sepsis et identifier les patients présentant des issues défavorables. ${ }^{11}$

Un système MEWS standardisé, tout comme les trousses de sécurité pour les patientes obstétricales, fournit un cadre fondé sur des données probantes pour guider les cliniciens dans la prise en charge sécuritaire des patientes obstétricales. L'accent est mis sur la préparation (c.-à-d. évaluation des risques, réalisation d'exercices in situ et de protocoles spécifiques à la condition, et élaboration de systèmes pour répondre aux signes avant-coureurs), la réaction adaptée et la communication des événements maternels imminents, ainsi que sur le désir de toujours améliorer le système lui-même. ${ }^{12}$ Un tel cadre peut être adapté tant à des contextes de ressources élevées que faibles. Les hôpitaux à faibles ressources peuvent mettre en œuvre les composantes réalisables et instaurer des processus pour le transfert approprié des patientes vers des centres bénéficiant de ressources supplémentaires au besoin.

L'utilisation adéquate des ressources, un triage approprié des patientes en fonction de leurs besoins cliniques et, par conséquent, la prestation de soins hospitaliers optimaux, sont essentiels pour améliorer les issues maternelles et réduire les disparités en matière de santé maternelle. En 2015, l'American College of Obstetricians and Gynecologists (ACOG) et la Society for Maternal-Fetal Medicine ont proposé un système de classification des hôpitaux obstétricaux fondé sur la disponibilité du personnel clinique et des ressources pour faciliter le triage des patientes en fonction du risque de morbidité et de mortalité maternelles. ${ }^{13}$ Dans un examen de la Base de données d'État de 2014 sur les patients hospitalisés tirée du Projet sur les coûts et l'utilisation des soins de santé et de l'Enquête annuelle de l'Association des hôpitaux américains, qui portait sur la situation dans sept états, $2,4 \%$ des femmes ont accouché dans des hôpitaux ne disposant pas du niveau approprié de ressources en santé maternelle, et $43 \%$ des femmes nécessitant un centre de niveau III ou IV ont accouché dans un centre inapproprié, ce qui pourrait inclure des services d'anesthésie mal équipés. ${ }^{14}$ Pour satisfaire les exigences de l'ACOG pour être considéré un centre de soins de niveau III (soins de surspécialité) et de niveau IV (centres périnataux régionaux), des « anesthésiologistes certifiés ayant une formation ou une expérience spéciale en 
obstétrique et responsables du service d'anesthésie obstétricale » sont nécessaires, et des services d'anesthésie doivent être disponibles en tout temps.

Pour combler les lacunes dans les domaines perçus d'amélioration de la qualité, la Society of Obstetric Anesthesia and Perinatology a élaboré un programme de désignation de Centres d'excellence pour les soins d'anesthésie obstétricale. Cette désignation vise à reconnaître les établissements et les programmes qui font preuve d'excellence en anesthésie obstétricale et qui fournissent une mesure élargie de la qualité des établissements offrant des soins d'anesthésie obstétricale. ${ }^{15}$ Cette désignation garantit que ces centres disposent de politiques en matière de dotation de personnel, de matériel, de protocoles et de politiques adaptés pour faire face aux urgences obstétricales et proposent des formations en simulation et d'équipe, une assurance de la qualité et des systèmes de suivi des patients.

Le rôle élargi des anesthésiologistes qui s'occupent des femmes pendant l'accouchement n'aurait pas pu être plus évident qu'au cours des 18 derniers mois, pendant la pandémie de COVID-19. Notre participation à une approche multidisciplinaire de l'élaboration de protocoles pour une prise en charge sécuritaire des patientes obstétricales atteintes de COVID-19 est devenue cruciale. Les anesthésiologistes ont été à l'avant-garde de l'élaboration de protocoles pour traiter les patientes infectées et non infectées, en tenant compte de l'administration de l'analgésie obstétricale, de l'anesthésie pour l'accouchement pas césarienne et de la prise en charge de toute complication, y compris le sepsis ou l'hémorragie du post-partum. Les anesthésiologistes obstétricaux disposaient d'un éventail unique de compétences pour fournir des soins sécuritaires et optimaux pendant la pandémie. ${ }^{16}$ Leur expertise en matière de prise en charge des voies aériennes et de l'hémodynamie, de dégagement des voies aériennes et de positionnement d'autres dispositifs de monitorage invasifs, ainsi que de l'administration de transfusions sanguines et d'autres interventions vitales est primordiale pour réduire la mortalité et la morbidité maternelles. La coordination des soins et l'élaboration des protocoles en collaboration avec des obstétriciens, des néonatologistes, des experts en soins intensifs et en maladies infectieuses ainsi qu'avec les services de santé environnementale, permettent à l'équipe multidisciplinaire de fournir des soins cliniques optimaux et sécuritaires aux patientes enceintes tout en protégeant les travailleurs de la santé. ${ }^{17}$

En résumé, le rôle des anesthésiologistes obstétricaux continue de s'étendre au-delà de la péridurale pour le travail et de l'anesthésie pour l'accouchement par césarienne. Pour prendre soin de nouvelles vagues de patientes obstétricales ayant des antécédents médicaux plus complexes, les anesthésiologistes doivent être plus que de simples consultants pendant le processus de travail obstétrical et d'accouchement.
L'époque de la répétition monotone de placements de cathéters pour l'anesthésie neuraxiale est révolue. Des évaluations critiques en temps opportun et les collaborations continues avec les autres membres de l'équipe de soins péripartum sont non seulement recommandées, mais également nécessaires pour que les anesthésiologistes deviennent de véritables médecins du péripartum. Nous devrions aspirer à devenir des membres dévoués de l'équipe pour optimiser les soins aux patientes enceintes tout au long de la période péripartum.

Disclosures Dr. George is a consultant for an Octapharma USA, Inc. Advisory Board and an Associate Editor of the Canadian Journal of Anesthesia.

\section{Funding statement None.}

Editorial responsibility This submission was handled by Dr. Philip M. Jones, Deputy Editor-in-Chief, Canadian Journal of Anesthesia.

Déclaration Le Dr George est consultant pour un Conseil consultatif d'Octapharma USA, Inc. et rédacteur adjoint du Journal canadien d'anesthésie.

\section{Déclaration de financement Aucune.}

Responsabilité éditoriale Cet article a été traité par Dr Philip M. Jones, rédacteur en chef adjoint, Journal canadien d'anesthésie.

\section{References}

1. Hawkins JL, Chang J, Palmer SK, Gibbs CP, Callaghan WM. Anesthesia-related maternal mortality in the United States. Obstet Anesth Dig 2012; 32: 1-2.

2. Hawkins JL, Koonin LM, Palmer SK, Gibbs CP. Anesthesiarelated deaths during obstetric delivery in the United States, 1979-1990. Anesthesiology 1997; 86: 277-84.

3. Cantwell R, Clutton-Brock T, Cooper G, et al. Saving Mothers' Lives: Reviewing maternal deaths to make motherhood safer: 2006-2008. The eighth report of the Confidential Enquiries into Maternal Deaths in the United Kingdom. BJOG 2011; 118 Suppl 1: 1-203.

4. Munro A, George RB. The peripartum anesthesiologist. Can J Anesth 2014; 62: 237-40.

5. Knight M, Bunch K, Tuffnell D, et al. Saving Lives, Improving Mothers' Care - Lessons learned to inform maternity care from the UK and Ireland Confidential Enquiries into Maternal Deaths and Morbidity 2015-17. Available from URL: https://www.npeu. ox.ac.uk/assets/downloads/mbrrace-uk/reports/MBRRACE-UK\% 20Maternal\%20Report\%202019\%20-\%20WEB\%20VERSION. pdf (accessed November 2021).

6. Balki I, Baghirzada L, Walker A, Lapinsky S, Balki M. Incidence, morbidity, and associated factors for sepsis in women hospitalized for delivery: a nationwide retrospective observational population-based study in Canada. Can J Anesth 2022; https://doi.org/10.1007/s12630-021-02158-0

7. World Health Organization. Trends in Maternal Mortality 2000 to 2017: Estimates by WHO, UNICEF, UNFPA, World Bank Group and the United Nations Population Division: executive summary; 2019. Available from URL: https://apps.who.int/iris/handle/ 10665/327596 (accessed November 2021). 
8. Bauer ME, Bateman BT, Bauer ST, Shanks AM, Mhyre JM. Maternal sepsis mortality and morbidity during hospitalization for delivery: temporal trends and independent associations for severe sepsis. Anesth Analg 2013; 117: 944-50.

9. Cordioli RL, Cordioli E, Negrini R, Silva E. Sepsis and pregnancy: do we know how to treat this situation? Rev Bras Ter Intensiva 2013; DOI: https://doi.org/10.5935/0103-507X. 20130056.

10. Kumar A, Roberts D, Wood KE, et al. Duration of hypotension before initiation of effective antimicrobial therapy is the critical determinant of survival in human septic shock. Crit Care Med 2006; 34: 1589-96.

11. Bauer ME, Housey M, Bauer ST, et al. Risk factors, etiologies, and screening tools for sepsis in pregnant women: a multicenter case-control study. Anesth Analg 2019; DOI: https://doi.org/10. 1213/ANE.0000000000003709.

12. Bernstein PS, Martin JN Jr, Barton JR, et al. National partnership for maternal safety: consensus bundle on severe hypertension during pregnancy and the postpartum period. Anesth Analg 2017; 125: 540-7.
13. Anonymous. Levels of maternal care: obstetric care consensus no, 9. Obstet Gynecol 2019; 134: e41-55.

14. Easter SR, Robinson JN, Menard MK, et al. Potential effects of regionalized maternity care on U.S. hospitals. Obstet Gynecol 2019; 134: 545-52.

15. Carvalho B, Mhyre JM. Centers of excellence for anesthesia care of obstetric patients. Anesth Analg 2019; 128: 844-6.

16. Li Y, Ciampa EJ, Zucco L, et al. Adaptation of an obstetric anesthesia service for the severe acute respiratory syndrome coronavirus-2 pandemic: description of checklists, workflows, and development tools. Anesth Analg 2021; DOI: https://doi.org/ 10.1213/ANE.0000000000005256.

17. Bauer ME, Bernstein K, Dinges E, et al. Obstetric anesthesia during the COVID-19 pandemic. Anesth Analg 2020; DOI: https://doi.org/10.1213/ANE.0000000000004856.

Publisher's Note Springer Nature remains neutral with regard to jurisdictional claims in published maps and institutional affiliations. 